\title{
ESTRATIFICAÇÃO VOLUMÉTRICA POR CLASSES DE ESTOQUE EM UMA FLORESTA OMBRÓFILA DENSA, NO MUNICÍPIO DE ALMEIRIM, ESTADO DO PARÁ, BRASIL ${ }^{1}$
}

\author{
Agostinho Lopes de Souza², Rodrigo Marques de Medeiros³, Luiz Marcos Silva Matos ${ }^{4}$, Kátia Regina \\ Silva ${ }^{5}$, Perez Alves Corrêa ${ }^{5}$ e Felipe Nascimento de Faria ${ }^{5}$
}

\begin{abstract}
RESUMO - Este estudo teve como objetivos agrupar as Unidades de Trabalho (UT) da Unidade de Produção Anual (UPA) em classes homogêneas de estoque volumétrico e analisar a composição florística, a diversidade e as estruturas horizontal e diamétrica, por classe de estoque. Os dados procederam do inventário de prospecção, ou censo, com mapeamento de árvores pré-comerciais e potencialmente comerciais e foram disponibilizados, mediante convênio, pela Orsa Florestal. A área de estudo localiza-se no Município de Almeirim, Estado do Pará. No Censo foram consideradas 469 UTs (250 x $400 \mathrm{~m}$ ), perfazendo 4.690 ha e 191.640 árvores com $\mathrm{DAP} \geq 35,0 \mathrm{~cm}$. Foram utilizadas análises de agrupamento (método de Ward) e discriminante (método de Fisher) e obtidas três classes de estoque, ou classes de produtividade. A riqueza e diversidade de espécies arbóreas com DAP $\geq 35 \mathrm{~cm}$ da UPA, respectivamente, 540 espécies e 4,52 nats.indivíduo ${ }^{-1}$, foram muito elevadas, porém realísticas, por se tratar de um censo. A intensidade de corte pode ser ordenada pela capacidade produtiva de cada UT e, dessa forma, resultar em colheitas de mínimo impacto ambiental, se comparada com a máxima intensidade de corte de $30 \mathrm{~m}^{3} \cdot \mathrm{ha}^{-1}$ com ciclo de corte inicial de 35 anos para plano de manejo florestal sustentável pleno. As parcelas permanentes também puderam ser distribuídas por classes de produtividade, reduzindo custos e aumentando a precisão das estimativas de crescimento da floresta manejada.
\end{abstract}

Palavras-chave: Manejo Florestal; Floresta natural; Classe de estoque.

\section{VOLUMETRIC STRATIFICATION BY STOCK CLASS IN A DENSE RAIN FOREST, CITY OF ALMEIRIM, STATE OF PARA, BRAZIL}

\begin{abstract}
The objective of this study was to group the Work Units (UT) of the Annual Production Unit (UPA) into homogeneous classes of volumetric stock and to analyze the floristic composition, the diversity and the horizontal and diametric structures, by stock class. Data came from a full survey or census, with the mapping of precommercial and potentially commercial trees. These data were provided, through agreement, by Orsa Florestal. The studied area is located in the municipality of Almeirim, State of Para, Brazil. In the census, 469 UTs ( $250 \times 400 \mathrm{~m}$ ), totalizing 4,690 ha and 191,640 trees with DAP $\geq 35.0 \mathrm{~cm}$ were evaluated. The cluster (Ward method) and discriminant (Fisher method) analyses were used and three stock classes, or productivity classes, were obtained. The richness and diversity of the arboreous species with DAP $\geq 35.0$ cm of the UPA were 540 species and 4.52 nats.individual ${ }^{-1}$, respectively. These figures are very high but realistic because they were obtained from a census. The cutting intensity can be determined by the productive capacity of each UT and thus result in a harvest with minimum environmental impact, when compared to the maximum
\end{abstract}

\footnotetext{
${ }^{1}$ Recebido em 28.05.2013 aceito para publicação em 29.04.2014.

${ }^{2}$ Departamento de Engenharia Florestal, Universidade Federal de Viçosa, Viçosa, MG, Brasil. E-mail: <alsouza@ufv.br>.

${ }^{3}$ Programa de pós-Graduação em Ciência Florestal, Universidade Federal de Viçosa, UFV, Brasil. E-mail: <rodrigo41260@gmail.com>.

${ }^{4}$ Mestre em Ciência Florestal, Departamento de Engenharia Florestal, Universidade Federal de Viçosa, Viçosa, MG, Brasil. E-mail: <luizmarcos.matos@gmail.com>

${ }^{5}$ Orsa Florestal. E-mail: <krsilva@grupoorsa.com.br>, <pacorrea@orsaflorestal.com.br>e <fnfaria@orsaflorestal.com.br>.
} 
cutting intensity of $30 \mathrm{~m}^{3}$.ha-1 with an initial cutting cycle of 35 years for a sustainable forest management plan. The permanent plots could also be distributed by productivity classes, reducing cost and increasing the growth estimate accuracies of the managed forest.

Keywords: Forest Management; Natural Forest; Stock Class.

\section{INTRODUÇÃO}

O interesse de consumidores pela madeira legal, sustentável e certificada, é cada vez maior. Então, para assegurar a gestão dos recursos florestais naturais em bases sustentáveis, a International Tropical Timber Organization (ITTO), World Wildlife Found (WWF), Center for International Forest Research (CIFOR) e Forest Stewardship Council (FSC), principalmente, estabeleceram diretrizes que visam, mediante um processo contínuo, ao desenvolvimento do manejo florestal sustentável.

No Brasil, o plano de manejo florestal sustentável (PMFS) é o instrumento básico para gestão sustentável das florestas (SFB, 2010). A vocação natural e legal para a produção florestal no bioma Amazônia (BRASIL, 2007, 2009), com cerca de 354.626.516 ha de florestas naturais, é o manejo florestal sustentável, visto que, além de 10,9 milhões de ha de unidades de conservação de uso sustentável, têm que ser mantidos, a título de reserva legal, $80 \%$ da área total de cada propriedade privada (BRASIL, 2011, 2012).

Enquanto os estoques mundiais de madeira de florestas nativas estão em declínio (CLEMENT; HIGUCHI, 2006; FRA, 2010), os estoques de madeira de florestas plantadas estão crescendo (FRA, 2010). Decresce a demanda por produtos florestais madeireiros e não madeireiros sem origem legal e insustentável e cresce a adesão de consumidores por produtos legal, sustentável e, sobretudo, com certificação de manejo e de cadeia de custódia (ADEODATO et al., 2011; BRASIL, 2011; FRA, 2010). Em 2009, foram identificadas 2.226 empresas madeireiras em funcionamento na Amazônia Legal, cuja extração madeireira legal totalizou cerca de 14,2 milhões de metros cúbicos de tora nativa, o que equivale a 5,8 milhões de metros cúbicos de madeira processada (SFB, 2010; IMAZOM, 2010). Cresceu também o número de empresas que produzem madeira certificada (CARNEIRO, 2006; ADEODATO et al., 2011). Até o final de setembro de 2010 havia, no Brasil, 592 certificações de cadeia de custódia para produtos de origem florestal e 74 certificações combinadas de manejo florestal com cadeia de custódia pelo FSC, o que perfazia 6.247.759,73 ha de florestas, sendo 2.737.221,57 ha de florestas nativas e 3.510.538,16 ha de florestas plantadas (SFB, 2010; BRASIL, 2011).

A empresa Orsa Florestal, que executa um PMFS pleno (BRASIL, 2006, 2009), numa área de reserva legal de aproximadamente 545 mil hectares com predomínio de Floresta Ombrófila Densa primária, no Estado do Pará, Brasil, é um desses produtores de madeira legal, sustentável e certificada, cujas experiências devem ser divulgadas.

Nas normas técnicas e legais dos PMFS da Amazônia consta o inventário $100 \%$, ou censo com mapeamento de árvores (MACHADO, 1988; AMARAL, 1998; FREITAS et al., 2005), que é fundamental para realizar a colheita de baixo impacto (HENDRISON, 1990; CARNEIRO, 2006; MAZZEI et al., 2010; MILLER et al., 2011).

Este trabalho teve como objetivos agrupar as unidades de trabalho da unidade de produção anual em classes homogêneas de estoque volumétrico e analisar a composição florística, estrutura horizontal e estrutura diamétrica, respectivamente, por classe de estoque.

\section{MATERIAL E MÉTODOS}

\subsection{Caracterização da área de estudo}

Os dados utilizados neste estudo foram disponibilizados mediante convênio celebrado entre a Orsa Florestal e a Universidade Federal de Viçosa (UFV). O estudo foi realizado na unidade 2 de produção anual (UPA-02) da unidade de manejo florestal (UMF) da Orsa Florestal, pertencente à empresa Jari Celulose S.A. Aárea de manejo florestal (AMF) da Orsa Florestal localiza-se em Monte Dourado, no Município de

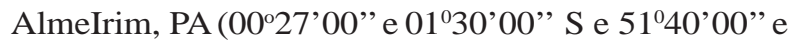
530200”' W), e soma aproximadamente 545 mil hectares. Os solos são Latossolos Amarelos, e o relevo é plano, com inclinação máxima de até $10 \%$. O clima na região caracteriza-se por duas estações bem distintas: uma 
chuvosa, de janeiro a julho, e outra seca, de agosto a dezembro. Segundo a classificação de Köppen, o clima é do subtipo Amw', quente, úmido e o mais frio dessa categoria. A temperatura permanece estável durante o ano inteiro, situando-se na média mensal de 25,5 a $27,4^{\circ} \mathrm{C}$. Predomina na UPA-02 a tipologia de Floresta Ombrófila Densa primária (VELOSO et al., 1991), sempre verde, sem estacionalidade marcante, cujos tipos florestais são caracterizados pela presença de fanerófitos, meso e macrofanerófitos, com aproximadamente 500 árvores por hectare e com DAP $\geq 10 \mathrm{~cm}$, perfazendo até $40 \mathrm{~m}^{2}$.ha-1 e $500 \mathrm{~m}^{3} \cdot \mathrm{ha}^{-1}$ (PIRES, 1974).

\subsection{Coleta dos dados}

Para execução do plano do manejo florestal, a empresa Orsa Florestal realizou um inventário de prospecção (censo), a fim de quantificar o volume de madeira por espécie e por área, a relação das espécies existentes, o número de árvores ou fustes, a qualidade da madeira e a localização das árvores. A AMF foi subdividida em 30 UPAs e estas, em unidades de trabalho (UTs). O número de UTs varia de acordo com o tamanho da UPA. As UTs perfazem, em média, 10 ha cada uma, com 250 m no sentido norte-sul e 400 $m$ no sentido leste-oeste. Para facilitar a operacionalidade do censo com mapeamento, cada UT foi subdividida em oito faixas de $250 \mathrm{~m} \mathrm{x} 50 \mathrm{~m}$, totalizando 1,25 ha cada uma.

No censo, mensuraram-se árvores com DAP $\geq 35,0$ cm de espécies de valor comercial (alto valor; bom valor; potencial; e sem valor) e árvores-matriz. Coletaram-se, ainda, os seguintes dados: número da árvore, coordenadas $(x, y)$ para localização, identificação botânica da espécie, circunferência à altura do peito (CAP), altura comercial (HT) e qualidade do fuste.

\subsection{Análises dos dados}

\subsubsection{Análise multivariada}

A análise dos dados consistiu, inicialmente, na quantificação do número de árvores, da área basal e do volume de cada uma das 469 UTs da UPA-02. De posse dessas variáveis de povoamento, procedeu-se à elaboração de uma matriz $X$ de dados, em que cada variável $x_{i j}$ representa o $j$-ésimo número de árvores $(j=1)$, área basal $(j=2)$ e volume $(j=3)$ na $i$-ésima UT, para $i=1,2, \ldots 469$.
Para agrupar as UTs em classes de produtividade (BRASIL, 2006, 2009), foram utilizados a distância euclidiana simples e o método de Ward e discriminante linear de Fisher (JOHNSON; WICHERN, 1988; SOUZA, 1989; SOUZA, 1989; SOUZA et al., 1990; SOUZA, 2006). Essas análises foram processadas por meio do software Statistica (STATSOFT INC., 2007).

A definição das classes de estoque se deu ao traçar a linha de corte (linha fenon) no dendrograma (SOUZA et al., 1990). Verificaram-se, por meio da análise discriminante, as probabilidades de classificação das UTs em classes de estoque volumétrico.

\subsubsection{Composição florística, diversidade e estrutura}

Para cada classe de estoque foram realizadas análises de riqueza e diversidade de espécies, estrutura horizontal e estrutura paramétrica por classes de diâmetros e de estoque.

A diversidade de espécies foi analisada pelos índices de Shannon ( $\left.H^{\prime}\right)$, equabilidade de Pielou ( $J$ e dominância de Simpson (C) (BROWER; ZAR, 1984; MAGURRAN, 1988). Para analisar a estrutura horizontal, utilizaramse os parâmetros fitossociológicos (MUELLERDUMBOIS; ELLENBERG, 1974): densidade absoluta $(D A$, em n.ha-1), densidade relativa $(D R)$, dominância absoluta ( $\left.D o A, \mathrm{em} \mathrm{m}^{2} \cdot \mathrm{ha}^{-1}\right)$, dominância relativa $(D o R)$, frequência absoluta $(F A)$, frequência relativa $(F R)$, valor de cobertura (VC\%) e valor de importância (VI\%). A estrutura paramétrica por classes de $D A P$ englobou o número de árvores (n.ha-1), a área basal $\left(\mathrm{m}^{2}\right.$.ha-1 $)$ e o volume $\left(\mathrm{m}^{3} \cdot \mathrm{ha}^{-1}\right)$ por grupos de espécies de valor comercial (alto valor, bom valor, potencial e sem valor), respectivamente, por classe de estoque ou produtividade (SOUZA; SOUZA, 2006).

O volume comercial por árvore foi estimado mediante o emprego da seguinte equação volumétrica: $V_{C}=$ $7,85398 \cdot 10^{-5} . D^{2} . H C . F a_{j} . F f$

em que:

$V c=$ volume de madeira comercial, $\mathrm{em}^{3}$;

$D=$ diâmetro à altura do peito $(D A P)$, em cm;

$H c=$ altura comercial, em m; e

$F a_{j}=$ fator de aproveitamento do tronco, em que $F a_{1}=1,0 ; F a_{2}=0,7 ; F a_{3}=0,3 ; F a_{4}=0,0$; e $F f=$ fator de forma da tora igual a 0,7 .

Revista Árvore, Viçosa-MG, v.38, n.3, p.533-541, 2014 


\section{RESULTADOS}

\subsection{Análises de agrupamento e discriminante}

Com base nas variáveis número de árvores, área basal e volume, a análise de agrupamento reuniu as 469 UTs da UPA-02 em três classes de produtividade ou classes de estoque (Tabela 1), e a análise discriminante linear de Fisher evidenciou que 96\% delas foram corretamente classificadas. Cada UT erroneamente classificada foi realocada na classe com maior probabilidade de pertencer, e novamente foi realizada a análise discriminante, para finalmente obter 98,7\% das UTs corretamente classificadas (Tabela 2).

\subsection{Composição florística e diversidade}

No censo com mapeamento das árvores com $\mathrm{DAP} \geq 35,0 \mathrm{~cm}$ foram contabilizadas 191.640 árvores, distribuídas em 540 espécies e 56 famílias, na área mapeada de 4.690 ha de Floresta Ombrófila Densa primária de terra-firme.

Na análise realizada por classe de estoque, foram obtidos os seguintes resultados: na classe I, foram reunidas 228 UTs contendo 122.784 árvores pertencentes a 491 espécies de 52 famílias; na classe II, 131 Uts, contabilizando 50.429 árvores pertencentes a 420 espécies de 52 famílias; e na classe III, 110 Uts, perfazendo 18.427 árvores pertencentes a 334 espécies de 51 famílias. Para a população com DAP $\geq 35 \mathrm{~cm}$, as diversidades estimadas pelo índice de Shannon (H', em nats.indivíduo $\left.{ }^{-1}\right)$, nas classes I, II e III, foram, respectivamente, 4,51; 4,52; e 4,47 (Tabela 3).

\subsection{Estrutura horizontal}

$\mathrm{Na}$ classe I de estoque, foram inventariadas 53,85 árvores.ha ${ }^{-1}$, que perfizeram $10,83 \mathrm{~m}^{2} . \mathrm{ha}^{-1}$ e 79,68 $\mathrm{m}^{3} \cdot \mathrm{ha}^{-1}$, sendo $46,89 \mathrm{~m}^{3} \cdot \mathrm{ha}^{-1}$ de alto valor comercial e

Tabela 1 - Número de unidades de trabalho (N.UTs), número de árvores do censo (N) e volumes (mínimo, médio, máximo, total e desvio-padrão) por classe de estoque, sendo cada UT de 10 ha.

Table 1 - Number of Work Units (N.UTs), number of trees from the census (N) and volumes (minimum, medium, maximum, total and standard deviation) by stock class, each work unit has 10 ha.

\begin{tabular}{ccccccc}
\hline Classe de & N.UTs & $\mathrm{N}$ & \multicolumn{3}{c}{ Volume $\left(\mathrm{m}^{3}\right)$} & \multicolumn{2}{c}{ Desvio-padrão } \\
\cline { 4 - 6 } estoque & & & Mínimo & Médio & Máximo & Total \\
\hline I & 228 & 122.784 & 627,07 & 796,80 & $1.181,12$ & $181.669,66$ \\
II & 131 & 50.429 & 384,47 & 545,49 & 663,25 & $71.458,81$ \\
III & 110 & 18.427 & 21,26 & 243,02 & 456,31 & $26.732,39$ \\
Total & 469 & 191.640 & 21,26 & 596,72 & $1.181,12$ & $279.860,87$ \\
\hline
\end{tabular}

Tabela 2 - Resultado final da análise discriminante mostrando as classificações corretas (\%) e as probabilidades ( $p$ ) de classificações das UTs nas classes de estoque.

Table 2 - Final result of the discriminant analysis showing the correct classifications (\%) and the classification probabilities (p) of the UTs in the stock classes.

\begin{tabular}{|c|c|c|c|c|c|}
\hline \multirow{2}{*}{$\begin{array}{l}\text { Classe de } \\
\text { estoque }\end{array}$} & \multicolumn{5}{|c|}{ Classificações } \\
\hline & I $(p=0,48614)$ & II $(p=0,27932)$ & III $(p=0,23454)$ & Classificação correta (\%) & Total \\
\hline I & 228 & 0 & 0 & 100,0 & 228 \\
\hline II & 3 & 128 & 0 & 97,7 & 131 \\
\hline III & 0 & 3 & 107 & 97,3 & 110 \\
\hline Total & 231 & 131 & 107 & 98,7 & 469 \\
\hline
\end{tabular}

Tabela 3 - Estimativas do índice de diversidade de Shannon ( $H^{\prime}$ '), equabilidade de Pielou $(J)$, dominância de Simpson $(D)$, coeficiente de mistura $(Q M)$ e número de árvores e de espécies $(S)$ por classe de estoque.

Table 3 - Estimates of the Shannon diversity index $\left(H^{\prime}\right)$, Pielou equability $(J)$, Simpson dominance $(D)$, mixture coefficient (QM) and number of trees and of species (S) by stock class.

\begin{tabular}{ccccrrrr}
\hline Classe de estoque & N $^{\circ}$ árvores (\%) & S & QM & H' & In(S) & J \\
\hline I & $122.784(64,1)$ & 491 & 250 & 4,51 & 6,20 & 0,73 & 0,98 \\
II & $50.429(26,3)$ & 420 & 120 & 4,52 & 6,04 & 0,75 \\
III & $18.427(9,6)$ & 334 & 55 & 4,47 & 5,81 & 0,77 \\
Total & 191.640 & 540 & 355 & 4,52 & 6,29 & 0,72 & 0,98 \\
\hline
\end{tabular}

Revista Árvore, Viçosa-MG, v.38, n.3, p.533-541, 2014 
9,13 $\mathrm{m}^{3} \cdot \mathrm{ha}^{-1}$ de bom valor comercial. Na classe II de estoque, foram inventariadas 38,50 árvores.ha $a^{-1}$, que totalizaram 7,52 $\mathrm{m}^{2}$. ha-1 e $54,55 \mathrm{~m}^{3}$.ha-1, sendo $31,60 \mathrm{~m}^{3} \cdot \mathrm{ha}^{-1}$ de alto valor comercial e $5,91 \mathrm{~m}^{3} \cdot \mathrm{ha}^{-1}$ de bom valor comercial. Na classe III de estoque, foram inventariadas 16,75 árvores.ha-1, que contribuíram com 3,34 $\mathrm{m}^{2}$.ha ${ }^{-1}$ e 24,30 $\mathrm{m}^{3}$.ha ${ }^{-1}$, sendo $14,30 \mathrm{~m}^{3} \cdot \mathrm{ha}^{-1}$ de alto valor comercial e 2,89 $\mathrm{m}^{3} \cdot \mathrm{ha}^{-1}$ de bom valor comercial.

As 10 espécies de maior VI\% na classe I foram: Dinizia excelsa (5,0\%), Vouacapoua americana (3,6\%), Manilkara bidentata (3,3\%), Licania micrantha (3,1\%), Goupia glabra (3,0\%), Parinari excelsa (2,9\%), Qualea paraensis (2,5\%), Tachigali myrmecophila (2,5\%), Manilkara huberi $(2,0 \%)$ e Minquartia guianensis, perfazendo 38,1\% da DR, $42,5 \%$ da DoR, 8,8\% da FR, 40,3\% do VC\% e 29,8\% do VI\%.

Na classe II de estoque, as 10 espécies de maior VI\% foram: Dinizia excelsa (3,9\%), Licania micrantha (3,4\%), Vouacapoua americana (3,3\%), Manilkara bidentata (3,3\%), Parinari excelsa (3,2\%), Goupia glabra (3,1\%), Qualea paraensis (2,9\%), Tachigali myrmecophila (2,5\%), Minquartia guianensis (1,95\%) e Qualea albiflora (1,91\%), que totalizaram 29,5\% do VI (\%), 37,6\% da DR, 40,8\% da DoR, 10,0\% da FR, $39,2 \%$ do VC\% e $29,5 \%$ do VI\%.

As 10 espécies de maiores VI\% na classe III de estoque, foram: Vouacapoua americana (5,7\%), Dinizia excelsa (4,1\%), Manilkara bidentata (3,5\%), Parinari excelsa (3,3\%), Licania micrantha (3,1\%), Goupia glabra (2,9\%), Tachigali myrmecophila (2,7\%), Qualea paraensis (2,7\%), Minquartia guianensis (2,2\%) e Manilkara huberi (2,0\%), que perfizeram 39,8\% da DR, $42,6 \%$ da DoR, $14,5 \%$ da FR, $41,2 \%$ do VC\% e 32,3\% do VI\%.

\subsection{Estrutura diamétrica}

As distribuições da DA (árvores.ha-1) e do volume comercial $\left(\mathrm{m}^{3} \cdot \mathrm{ha}^{-1}\right)$, por classes de DAP e de estoque encontram-se nas Figuras 1 e 2, respectivamente. $\mathrm{Na}$ classe I, as árvores pré-comerciais ou de futuro $(35,0 \leq$ DAP $<50,0 \mathrm{~cm}$ ) perfizeram 36,80 árvores.ha-1 (68,3\%), 4,87 m².ha-1 (45,0\%) e 33,90 m³.ha-1 $(42,6 \%)$, enquanto as de futuro (DAP $\geq 50,0 \mathrm{~cm}$ ) somaram 17,05 árvores.ha-1 (31,7\%), 5,95 m².ha-1 (55,0\%) e $45,775 \mathrm{~m}^{3} \cdot \mathrm{ha}^{-1}(57,4 \%)$.
Na classe II de estoque, as árvores pré-comerciais (35,0 $\leq$ DAP $<50,0 \mathrm{~cm})$ somaram 26,72 árvores.ha ${ }^{-1}$ (69,4\%), $3,55 \mathrm{~m}^{2} \cdot$ ha $^{-1}(47,2 \%)$ e $24,31 \mathrm{~m}^{3} \cdot \mathrm{ha}^{-1}$ (44,6\%), enquanto as árvores potencialmente comerciais (DAP $\geq 50,0 \mathrm{~cm}$ ) perfizeram 11,78 árvores.ha-1 (30,6\%), 3,97 m².ha-1 $(52,8 \%)$ e $30,24 \mathrm{~m}^{3} \cdot \mathrm{ha}^{-1}(55,4 \%)$.

Na classe III de estoque, as árvores pré-comerciais $(35,0 \leq$ DAP $<50,0 \mathrm{~cm})$ totalizaram 11,35 árvores.ha ${ }^{-1}$

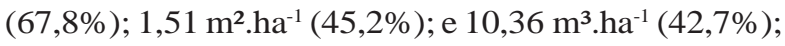
e as árvores potencialmente comerciais (DAP $\geq 50,0 \mathrm{~cm}$ ), 5,40 árvores.ha-1 (32,2\%), 1,83 $\mathrm{m}^{2} \cdot \mathrm{ha}^{-1}$ (54,8\%) e $13,94 \mathrm{~m}^{3} \cdot \mathrm{ha}^{-1}(57,3 \%)$.

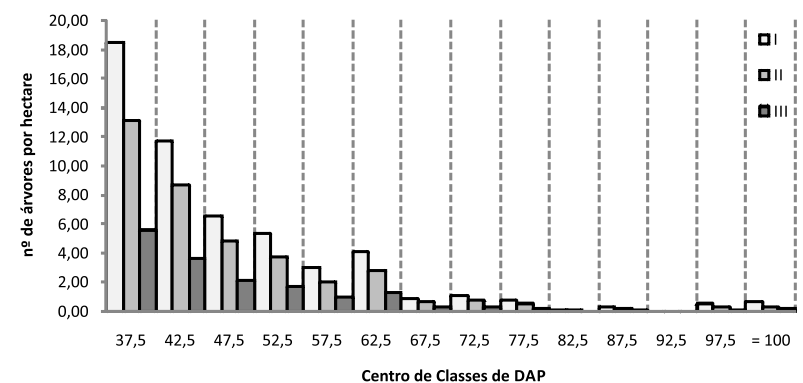

Figura 1 - Número de árvores.ha ${ }^{-1}$ por classe de DAP e por classe de estoque (I, II e III), UPA-02, empresa Orsa Florestal, Município de Almerim, Estado do Pará.

Figure 1 - Number of trees. ha $a^{-1}$ by DAP class and by stock class (I, II and III), UPA-02, Orsa Florestal company, city of Almeirim, State of Para, Brazil.

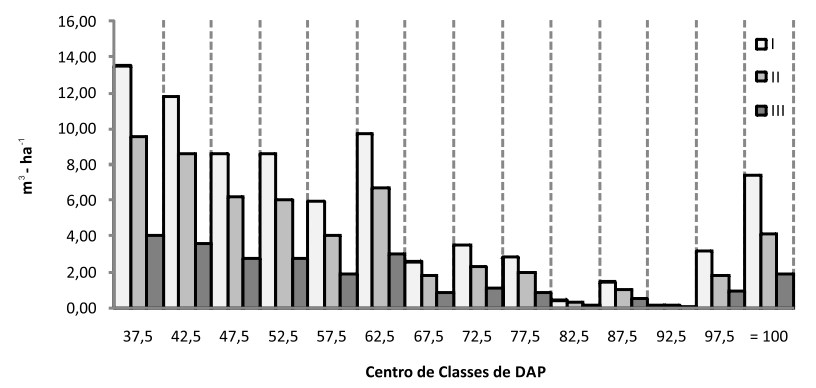

Figura 2 - Volume comercial $\left(\mathrm{m}^{3} \cdot \mathrm{ha}^{-1}\right)$ por classe de DAP e por classe de estoque (I, II e III), UPA-02, empresa Orsa Florestal, Município de Almeirim, Estado do Pará.

Figure 2 - Commercial volume $\left(\mathrm{m}^{3} . \mathrm{ha}^{-1}\right)$ by DAP class and stock class (I, III and III), UPA-02, Orsa Florestal company, city of Almeirim, State of Para, Brazil.

Revista Árvore, Viçosa-MG, v.38, n.3, p.533-541, 2014

\section{Silf}




\section{DISCUSSÃO}

\subsection{Análises de agrupamento e discriminante}

As classes de estoque (Tabela 1) foram nomeadas em ordem decrescente de volume médio observado, em que a classe I foi a de maior volume médio, a classe II apresentou volume médio intermediário e a classe III, a de menor volume médio (Tabela 1). Assim, essas classes agruparam UTs homogêneas em termos dos parâmetros volume, área basal e número de árvores de todas as espécies ocorrentes na UPA-02. Portanto, as UTs com alta, média e baixa produtividade correspondem, respectivamente, às classes I, II e III de estoque.

A fim de verificar a consistência da classificação das UTs nos grupos formados a partir da análise de agrupamento, aplicou-se a análise discriminante linear de Fisher, cujo resultado demonstrou que $96 \%$ das UTs foram corretamente classificadas nas respectivas classes. Posteriormente, fundamentado na análise discriminante, as UTs erroneamente classificadas foram realocadas nas classes com maior probabilidade de pertencer, e, novamente, foi realizada a análise discriminante, para finalmente obter a classificação final com 99\% das UTs classificadas nos grupamentos com a mais alta probabilidade de pertencerem (Tabela 2).

Nota-se na Tabela 2 que o número de UTs por classe de estoque difere dos resultados apresentados na Tabela 1, demonstrando que a análise discriminante é utilizada não só para avaliar a consistência da análise de agrupamentos, mas também para alocar UTs nos grupos cuja probabilidade de pertencer é maior (JOHNSON; WICHERN, 1988).

Resultados semelhantes foram encontrados por Souza (1989), Souza et al. (1990) e Souza e Souza (2006), em termos do número de classes de estoque volumétrico, ao empregarem o método de Ward para formação dos agrupamentos em conjunto com a distância euclidiana simples, como medida de distância entre as UTs.

\subsection{Composição florística e diversidade}

Para a população total (DAP $\geq 35,0 \mathrm{~cm}$ ), a estimativa de diversidade pelo índice de Shannon (H’) foi de 4,52 e para as classes de estoque I, II e III, 4,51, 4,52; e 4,47, respectivamente (Tabela 3). Apesar de o nível de inclusão deste estudo ter sido maior, os valores de diversidade foram maiores em relação aos de estudos realizados em floresta tropical de terra-firme, na Amazônia (RIBEIRO et al., 1999; MACIEL et al., 2000; YARED et al., 2000; SOUZA, 2006; AZEVEDO, 2006). Como se depreende dos resultados, a elevada diversidade decorre mais da elevada riqueza e está associada, positivamente, com a produtividade da floresta (Tabelas 1 e 3$)$.

Já o índice de Pielou (J) não apresentou valores elevados porque, apesar da elevada riqueza (S), o número de árvores também foi muito elevado, mas o coeficiente de mistura (QM) foi muito alto, sobretudo nas classes de maior produtividade. Entretanto, retratam a realidade da floresta porque foram obtidos do censo com mapeamento de árvores (Tabela 3).

\subsection{Estrutura horizontal}

Em termos de DoR, as espécies seguiram ordenação semelhante à das espécies de maior VI\%, com destaque para Dinizia excelsa, que é de alto valor comercial, ocorre em todas as classes de estoque (Figuras 2, 3 e 4) e com árvores de grande diâmetro.

As espécies Goupia glabra, Manilkara bidentata, Parinari excelsa, Qualea paraensis, Licania micrantha, Tachigali alba e Brosimum parinarioides ocorreram em todas as 228 UTs da classe I de estoque. A espécie Vouacapoua americana apresentou maior DR e baixa FR, mesmo relacionada em todas as classes de produtividade e entre as de maior VI\%.

A espécie com maior DoR na classe II, Dinizia excelsa, seguiu a mesma tendência apresentada na classe I de estoque, com alta DoR e média DR. Considerando-se a FR, as espécies Manilkara bidentata, Goupia glabra e Qualea paraensis ocorreram em todas as 131 UTs da classe II, e as espécies Licania micrantha, Parinari excelsa e Brosimum parinarioides estavam presentes em 130 UTs cada uma. As espécies Vouacapoua americana, Dinizia excelsa e Manilkara bidentata compartilham o ranking de maior VI\%. Mas, em relação à FR, nenhuma espécie ocorreu em todas as 110 UTs da classe III, e somente as espécies Manilkara bidentata, Parinari excelsa e Goupia glabra estavam presentes em mais de 100 UTs (90\%).

\section{Estrutura diamétrica}

A distribuição da DA (árvores.ha ${ }^{-1}$ ) por classes de DAP e de estoque (Figura 1) seguiu a tendência típica de distribuição exponencial negativa, ou seja, em “J” 
invertido (HUSCH et al., 2003) e com elevados estoques de árvores com DAP iguais ou superiores ao diâmetro mínimo de corte (DMC) de 50,0 cm (BRASIL, 2006; 2009).

Verifica-se, na Figura 2, que os estoques volumétricos de árvores com DAP $\geq 50,0 \mathrm{~cm}$ nas classes I e II superam a intensidade máxima de corte de $30 \mathrm{~m}^{3}$.ha-1 (BRASIL, 2006; 2009). Mas, para que a colheita atenda às restrições técnica e legal (BRASIL, 2006; 2009) e seja de impacto reduzido, há rigorosa seleção de árvores para abate direcional não só para harmonizar a intensidade de corte com a produtividade da floresta, mas, sobretudo, para aumentar a segurança do trabalho e precaver-se de danos à madeira, às árvores-matriz, às árvores précomerciais e às espécies raras e em risco (BARLOW et al., 2010), enfim, à floresta remanescente (PUTZ et al., 2008; MAZEI et al., 2010). A Orsa Florestal promoveu rigorosa seleção de árvores para corte e diminuiu a extração de madeira para, em média, $22 \mathrm{~m}^{3}$.ha ${ }^{-1}$ e com melhor desempenho financeiro(REVISTAGLOBORURAL, 2012). Dessa forma, atendeu às restrições legais e ambientais, e adequou-se a parâmetros técnicos e econômicos e estabeleceu o equilíbrio entre a intensidade de corte, a produtividade da floresta e o ciclo de corte (BRASIL, 2006).

\section{CONCLUSÕES}

As análises de agrupamento (distância euclidiana e método de Ward) e discriminante (método de Fisher) possibilitaram estratificar extensa e heterogênea UPA em grupos de UTs homogêneas em termos de variáveis de povoamento ou de estoque comercial.

As classes de UTs homogêneas em estoque de árvores pré-comercial e potencialmente comercial são úteis para propósitos de determinação do corte e planejamento e realização de atividades de colheita, tratamentos silviculturais e monitoramento da floresta manejada para produção sustentável de madeira.

\section{AGRADECIMENTOS}

Ao Grupo Orsa, pela concessão dos dados mediante o convênio celebrado com a Sociedade de Investigações Florestais (SIF); e à Universidade Federal de Viçosa, com vigência de 15/01/08 a 14/01/2013.

\section{REFERÊNCIAS}

ADEODATO, S. et al. Madeira de ponta a ponta: o caminho desde a floresta até o consumo. São Paulo: FGV/RAE, 2011. 128p.
AMARAL, P. et al. Floresta para sempre: um manual para a produção de madeira na Amazônia. Belém: IMAZON, 1998. 137p.

AZEVEDO, C. P. Dinâmica de florestas submetidas a manejo na Amazônia Oriental: experimentação e simulação. 2006. 236f. Tese (Doutorado em Engenharia Florestal) - Universidade Federal do Paraná, Curitiba, 2006.

BARLOW, J. et al. Measuring the conservation value of tropical primary forests: The effect of occasional species on estimates of biodiversity uniqueness. Plos One, v. 5, n. 3, mar. 2010. Disponível em: <www.plosone.org>. Acesso em: 11 mar. 2013.

BRASIL. Instrução Normativa, No 5, de 11 dez. 2006. Dispõe sobre procedimentos técnicos para elaboração, apresentação, execução e avaliação técnica de Planos de Manejo Florestal Sustentável-PMFSs nas florestas primitivas e suas formas de sucessão na Amazônia Legal, e dá outras providências. Diário Oficial da União, Seção 1 , Brasília, 13 dez. 2006.

BRASIL. Ministério do Meio Ambiente. Instituto Brasileiro do Meio Ambiente e Recursos Naturais Renováveis IBAMA. Normas Florestais Federais para a Amazônia. Brasília: IBAMA, Diretoria de Uso Sustentável da Biodiversidade e Florestas, 2007. 176 p.

BRASIL. Conselho Nacional Do Meio AmbienteCONAMA. Resolução/CONAMA N 406, de 2 de fevereiro de 2009. Estabelece parâmetros técnicos a serem adotados na elaboração, apresentação, avaliação técnica e execução de Plano de Manejo Florestal Sustentável - PMFS com fins madeireiros, para florestas nativas e suas formas de sucessão no bioma Amazônia. Diário Oficial da União, Brasília, n. 26, 6 de fev. de 2009.

BRASIL. Ministério do Meio Ambiente. Serviço Florestal Brasileiro. Portaria n ${ }^{\circ} 75$, de 5 de agosto de 2011. Publica o resumo executivo do Plano Anual de Outorga Florestal - PAOF 2012. Diário Oficial da União, Brasília, seção 1, p.128, 08 de ago. 2011.

Revista Árvore, Viçosa-MG, v.38, n.3, p.533-541, 2014 
BRASIL. Lei No 12.651, de 25 de maio de 2012. Dispõe sobre a proteção da vegetação nativa; altera as Leis $\mathrm{n}^{\circ} \mathrm{s} 6.938$, de 31 de agosto de 1981, 9.393, de 19 de dezembro de 1996, e 11.428, de 22 de dezembro de 2006; revoga as Leis $n^{\circ} \mathrm{s} 4.771$, de 15 de setembro de 1965, e 7.754, de 14 de abril de 1989, e a Medida Provisória n 2.166- 67, de 24 de agosto de 2001; e dá outras providências.

Diário Oficial da União, Brasília, 28 de maio de 2012.

BROWER, J. E.; ZAR, J. H. Field and laboratory methods for general ecology. 2.ed. Iowa: Northern Illinois University, 1984. 226 p.

CARNEIRO, M. S. ONGs, expertise e o mercado do desenvolvimento sustentável: a certificação florestal na Amazônia brasileira. Novos

Cadernos NAEA, v.9, n.1, p.131-160, jun. 2006.

CLEMENT, C. R.; HIGUCHI, N. A floresta amazônica e o futuro do Brasil. Ciencia e . Cultura [online], v.58, n.3, p.44-49, 2006.

FRA Global Forest Resources Assessment 2010. Rome: FAO, 2010. 371 p. Disponível em: $<$ www.fao.org/forestry/fra/fra2010/en>. Acesso em: 29 nov. 2011.

FREITAS, L. J. M. et al. Análise técnica e estimativas de custos de inventário de prospecção em uma Floresta Estacional Semidecidual Submontana. Revista Árvore, v.29, n.1, p.65-75, 2005.

HENDRISON, J. Damage-controlled logging in managed rain forest in Suriname. The Netherlands: Wageningen Agriculture University, 1990. 204p.

HUSCH, B.; BEERS, T. W.; KERSHAW JR., J. A. Forest mensuration. 4.ed. New Jersey: John Wiley \& Sons, 2003. 443p.

INSTITUTO DO HOMEM E MEIO AMBIENTE DA AMAZÔNIA - IMAZON. Levantamento de iniciativas de Manejo Florestal Comunitário e Familiar na Amazônia Legal. Belém: IMAZON, 2010. 79p. (Relatório Técnico).

JOHnson, R. A.; WICHERn, D. W. Applied multivariate statistical analysis. New Jersey: Prentice Hall, 1988. 607p.

Revista Árvore, Viçosa-MG, v.38, n.3, p.533-541, 2014
MACHADO, S. A. Complete enumeration forest inventory versus cluster sampling method applied in the Amazonic rain forest. Revista Floresta, v.17, n.1/2, p.122-130, 1988.

MACIEL, M. N. M.; QUEIROZ, W. T.; OLIVEIRA, F. A. Parâmetros fitossociológicos de uma floresta tropical de terra firme na Floresta Nacional de Caxiuanã-PA. Revista Ciências Agrárias, n.34, p.85-106, 2000.

MAGURRAN, A. E. Ecological diversity and its measurement. New Jersey: Princen University Press, 1988. 179p.

MAZZEI, L. et al. Above-ground biomass dynamics after reduced-impact logging in the eastern Amazon. Forest Ecology and Management, v.259, n.3, p.367-373, 2010.

MILLER, S. et al. Reduced impact logging minimally alters tropical raiforest carbon and energy exchange. PNA Early Edition, p. 1-5. 2011. Acesso em: <http/www.pnas.org/cgi/doi/10.1073/ pnas.1105068108>.

MUELLER-DOMBOIS, D.; ELLENBERG, H. Aims and methods of vegetation ecology. New York: Jonh Wiley \& Sons, 1974. 547p.

PIRES, L. M. Tipos de vegetação da Amazônia. Boletim do Museu Paraense Emílio Goeldi, v.20, n.1, p.179-202, 1974.

PUTZ, F. E. et al. Reduced-impact logging: Challenges and opportunities. Forest Ecology and Management, v.256, n.7, p.1427-1433, 2008.

REVISTA GLOBO RURAL. Lucro verde na floresta, mar. 2012.

RIBEIRO, R. J. et al. Estudo fitossociológico nas regiões de Carajás e Marabá, PA, Brasil. Acta Amazônica, v.29, n.2, p.207-222, 1999.

SERVIÇO FLORESTAL BRASILEIRO - SFB. Florestas do Brasil em resumo - 2010: dados de 2005-2010. 152p. 
SOUZA, A. L. Análise multivariada para manejo de florestas naturais: alternativas de produção sustentada de madeiras para serraria. 1989. 255f. Tese (Doutorado em Ciência Florestal) - Universidade Federal do Paraná, Curitiba, 1989.

SOUZA, A. L. et al. Análises multivariadas para manejo de floresta natural na Reserva Florestal de Linhares, Espírito Santo: análises de agrupamento e discriminante. Revista Árvore, v.14, n.2, p.85-101, 1990.

SOUZA, A. L.; SOUZA, D. R. Análise multivariada para estratificação volumétrica de uma floresta ombrófila densa de terra-firme, Amazônia Oriental. Revista Árvore, v.30, n.1, p.49-54, 2006.
STATSOFT INC. Statistica data analysis system version 7.0. Tulsa: Statsoft, 2007.

VELOSO, H. P.; RANGEL FILHO, A. L. R.; LIMA, J. C. A. Classificação da vegetação brasileira, adaptada a um sistema universal. Rio de Janeiro: Departamento de Recursos Naturais e Estudos Ambientais Instituto Brasileiro de Geografia e Estatística, 1991.

YARED, J. A. G.; COUTO, L.; LEITE, H. G. Diversidade de espécies em florestas secundária e primária, sob efeitos de diferentes sistemas silviculturais, na Amazônia Oriental. Revista Árvore, v.24, n.1, p.83-90, 2000. 